\title{
Evaluating the effectiveness of Facebook to impact the knowledge of evidence-based employment practices by individuals with traumatic brain injury: A knowledge translation random control study
}

\author{
Katherine J. Inge ${ }^{\mathrm{a}, *}$, Carolyn W. Graham ${ }^{\mathrm{b}}$, James W. McLaughlin ${ }^{\mathrm{c}}$, Doug Erickson ${ }^{\mathrm{a}}$, \\ Paul Wehman ${ }^{\mathrm{a}}$ and Hannah E. Seward ${ }^{\mathrm{a}}$ \\ ${ }^{a}$ Rehabilitation Research and Training Center, Virginia Commonwealth University, Richmond, VA, USA \\ ${ }^{\mathrm{b}}$ Center for Rehabilitation Sciences and Engineering, Virginia Commonwealth University, Richmond, VA, USA \\ ${ }^{\mathrm{c}}$ NewFaze, Richmond, VA, USA
}

Received 5 April 2016

Accepted 21 September 2016

\begin{abstract}
.
BACKGROUND: Individuals with traumatic brain injury (TBI) experience difficulty with obtaining and maintaining employment post-injury. Although vocational rehabilitation (VR) can be one option to provide individuals with TBI support and services to lead to successful employment outcomes, information about these services can be difficult and confusing to navigate. Providing information on evidence-based employment practices to individuals with TBI through social media could be an effective approach.

OBJECTIVE: The objective of this study was to compare the effect of a knowledge translation (KT) strategy and the use of a secret Facebook group, on the knowledge of evidence-based employment research by individuals with traumatic brain injury (TBI).

METHODS: The study used a randomized pretest-posttest control group design. Sixty individuals with TBI were recruited through clubhouse programs in the state where the authors resided as well as through support groups nationally for individuals with TBI, and were randomly assigned to one of two groups. Both groups received information on evidence-based employment practices for individuals with traumatic brain injury (TBI) over a three month period. One group received the information via participation in a secret Facebook group while the comparison group received information as an "e-news" email blast. Participants were assessed pre- and post-intervention with a Likert-scale instrument designed to measure knowledge of evidenced-based employment information for TBI.

RESULTS: Both groups gained a significant amount of knowledge between baseline and post-intervention. However, there were no significant differences between groups in knowledge gained at post-intervention.

CONCLUSION: While the study did not identify the most effective means of delivering information to individuals with TBI, it does provide some guidance for future KT research.
\end{abstract}

Keywords: Social media, vocational rehabilitation, VR, supported employment, employment of people with disabilities

\footnotetext{
*Address for correspondence: Katherine J. Inge, PhD, OTR, Rehabilitation Research and Training Center, Virginia Common-
} 


\section{Background}

Traumatic brain injury (TBI) can result in a variety of cognitive deficits, impaired psychosocial functioning, and physical and/or sensory functioning [1]. As a result, individuals with TBI often experience difficulty becoming employed post-injury and maintaining employment for extended periods of time [1]. Return to work (RTW) rates are highly variable across the literature; however, Van Velzen, Van Bennekom, Edelaar, Sluiter, and Frings-Dresen reported in their systematic review of TBI studies that on average, approximately $40 \%$ of individuals who sustain a TBI have achieved RTW at both the one and two-year milestones [2]. In another paper, Shames, Treger, Ring, and Giaquinto discussed RTW as a complex interaction of variables, including pre-injury factors (age, education, employment history); injuryrelated factors (type, severity, location); post-injury impairments (cognitive, physical, behavioral, psychological); and personal and environment factors (marital status, alternative income sources, social support) [3]. Regardless of the strength of evidence of known risk factors, it would be a mistake to confuse the likelihood of RTW with inevitability, which could result in lower expectations, reduced rehabilitation efforts, and self-fulfilling prophecies for TBI survivors at high risk for poor post-injury employment outcomes.

Vocational rehabilitation (VR), through the use of the federal and state VR programs, is one option for individuals with TBI to obtain the needed rehabilitation supports that can lead to successful employment outcomes. Inge and colleagues reported on the number of individuals with TBI who exited services from this program as documented in the RSA-911 reports during fiscal years $2011-2013$ [4]. Across all three years, slightly less than one-third of the individuals with TBI exited the VR system successfully with an employment outcome. These data clearly point to the need for innovative approaches to assist individuals with TBI to enter or re-enter the workplace post injury. In addition, few individuals with TBI know about benefits, incentives, and eligibility for incentives for returning to work [5]. One such innovative approach is to provide information on evidence-based employment practices to individuals with TBI using social media.

Social media is an important networking and information dissemination tool with Facebook reported as the largest social network [6]. In the first quarter of 2015, Facebook reported that it had 1.44 billion monthly active users with approximately $65 \%$ of their members using the service daily [7]. By the fourth quarter of 2015, Facebook reported a record 1.591 billion active monthly users and 1.038 billion daily active users [6]. The length of time that users spend on Facebook daily has been estimated to be 20 minutes globally with 40 minutes reported as the average for users in the United States [8]. Pew Research Center reported that $19 \%$ of people who are employed use Facebook for work-related purposes. Of these workers who used Facebook, LinkedIn, and Twitter, 78\% said they use social media to network, find job opportunities, and finding information they need to do their work [9].

Individuals with disabilities are also users of Facebook. A reported 69\% of a sample of 172 respondents with varying physical and cognitive disabilities viewed Facebook a minimum of once daily while $44.2 \%$ logged up to 30 minutes per visit [10]. Tsaousides and colleagues found that $59.6 \%$ of a four state sample of 96 individuals with TBI also used Facebook regularly [11]. Furthermore, a total of $70 \%$ of these users reported that increased knowledge about Facebook would encourage them to use it even more often. The interactive nature of Facebook posts, comments, and replies allows for information to be shared along with asking questions or seeking clarification. Of those who were not users, about $50 \%$ expressed interest in learning how to navigate the social networking site. Steady use among individuals with disabilities including individuals with TBI, along with documented interest in Facebook by non-users, suggests that social networking could serve as a strong platform for delivering employment specific intervention information in a contemporary way.

As part of a larger study, the authors of this research conducted focus groups to determine how individuals with disabilities want to obtain evidence-based research on employment. Focus groups that included individuals with TBI were conducted to determine what they know about evidence-based research and how they access research information. The focus groups revealed the primary source of information for participants was a trusted expert, such as a VR Counselor, academic or career advisor, physician, or another and more knowledgeable individual with a disability. Participants also reported an increasing use of internet resources to obtain employment information, including websites, webinars, listservs, and social networking sites such as Facebook, LinkedIn, and Twitter. This information provided the foundation on which to design the current study to determine 
if social media, specifically Facebook, can be used as a knowledge translation (KT) strategy to deliver information on evidence-based employment research to individuals with disabilities specifically individuals with TBI. The research questions addressed included the following:

1. Is social media, specifically a "secret" Facebook group led by a peer mentor, effective in increasing the knowledge of evidence-based employment research for individuals with TBI?

2. What is the relationship between participant demographics and the effectiveness of the KT strategy to impact evidence-based employment knowledge and use by individuals with TBI?

\section{Method}

\subsection{Participants}

Participants were recruited from clubhouse programs within the state where the researchers resided, as well as through support groups nationally for individuals with TBI. In order to participate, individuals had to have a TBI, be a U.S. citizen, be between the ages of 18 and 65 years, and be able to provide informed consent. The target number for recruitment was a minimum of 60 participants to allow random assignment of 30 individuals with TBI into two groups.

A research assistant contacted the clubhouses and asked if a staff member from the study could visit and make a brief presentation on the research. Five clubhouses were visited within the state, and a brief presentation was made. The recruitment flyer was disseminated to the members that included the link to the online consent process. The research assistant left each clubhouse after the flyers were distributed to ensure that the members did not feel obligated to consent to participate. In addition, the research assistant conducted an online state-by-state search of brain injury support groups for individuals with TBI. Through email and phone contact, support groups were asked to disseminate the recruitment flyer. Using these two recruitment strategies, 138 individuals used the link in the recruitment flyer to complete the online consent process.

The online consent included a description of the study as well as information on confidentiality and protection of the participants' identity on Facebook. For the purpose of follow-up contact for the pretest and posttest, individuals provided their name, email, and mailing address during the consent process. All identifying information was stored in a password protected database that was accessible only to the study researchers. In addition, each participant was assigned a code number for storing pretest and posttest data so that individual names were not associated with the data for data analysis.

Of the 138 individuals who self-reported to have a traumatic brain injury and consented to participate, 71 individuals were withdrawn from the study who did not meet the participation criteria. Eleven participants were removed because they were not U.S. citizens, even though the consent process clearly stated that only U.S. citizens could participate. These individuals were thanked for their interest via email and told that the study was limited to residents of the United States. Six individuals were dropped from the study because the email addresses provided for follow-up contact were undeliverable. One person was removed from the study for sending sexually explicit emails to the lead researcher. One individual was not his own legal guardian and was removed. Three participants requested to be withdrawn upon follow-up contact asking them to complete the pretest. Forty-nine individuals were removed because they did not complete the pretest after three reminder emails requesting them to do so. After these individuals were withdrawn from the study, 67 individuals remained and were randomly assigned to one of the two groups with 34 individuals assigned to the Facebook intervention and 33 individuals assigned to the email brief intervention.

\subsection{Intervention and comparison conditions}

The KT strategy selected to deliver the information was a secret Facebook group with the comparison group receiving "e-news" email blasts. The "e-news" email blast was considered the "business as usual" approach to delivering information on employment evidence-based practices. The content for the email blasts was developed by the lead researcher based on evidence-based practices in supported employment for individuals with TBI. The same evidence-based information formed the foundation for the posts that were made in the secret Facebook group. The information provided included information concerning state resources, assistive technology, disclosure, and interviewing techniques. This information was presented in non-academic language to allow understanding by all participants and was from research 
data bases and developed research center materials. This was done to ensure that both groups received parallel information. Participants in the "e-news" group received one email per month during the three month time period. These included brief articles as well as links to websites with additional employment information on the topics.

To protect the confidentiality of the participants, a secret Facebook group was selected for the social media KT intervention strategy. During the consent process, the use of Facebook was described, and the participants were told that their names would be visible to the other participants. Facebook has policies regarding member access including a policy stating that a member can only have one account. Therefore, the researchers could not assign a code name to participants, nor could the members create a fictitious name. This information was clearly described to the participants during the consent process.

A secret group offers the highest privacy settings on Facebook to protect the confidentiality of the participants. The lead researcher served as the group administrator. Participants randomly assigned to this intervention were invited through Facebook by the administrator who then had to approve their requests to enter the group. Only the individuals invited by the administrator were able to join. In other words, group members could not invite people nor could other Facebook members request to join the group. A number of other security features ensured the confidentiality of the participants. For instance, other Facebook users could not access the group or see any of the information. A secret group does not show up on Facebook searches conducted by nonmembers. If someone has the URL to the secret group they cannot gain access without being invited to the group. If the secret group URL is posted in another secret group, the URL is still not visible or accessible. Finally, secret groups are not indexed by Google.

As previously stated, the lead researcher served as the administrator for the group as well as the content expert on employment evidence-based practices. Based on the focus group conducted prior to the study, the researchers also identified a peer mentor to serve as the group facilitator. This individual had experienced a TBI, had been a supported employment client, and had earned his master's degree in rehabilitation counseling post-injury. The lead researcher and the peer facilitator met once weekly to discuss the group postings and to plan for the information that would be shared in the coming week.

\subsection{Procedure}

The research was reviewed and approved by the Institutional Review Boards used by SEDL, the organization that received funding for the research and the authors' University. The intervention spanned a three month period of time and began after the 67 participants had been randomly assigned to one of the two groups. An "e-news" was sent to the participants in the email blast group using the contact database at the beginning of each of the three months. The participants could read the information at their convenience. Posts were made by the peer mentor and the lead researcher a minimum of five times a week during the work week. If participants posted during the weekend, the lead researcher was alerted through Facebook's notification feature that a post had been made. Typically, responses were posted within an hour but always within 24 hours. At the end of the three months, the participants were asked to complete the posttest. In total, 60 participants completed both the pre- and posttests. Of the 34 participants randomly assigned to the Facebook group, 30 completed the pre- and the posttest. Of the 33 participants in the brief group, 30 completed the pre- and the post-test. Participants were compensated in the form of a $\$ 25$ gift card that could be used at a retailer of their choice upon completion of the posttest.

\subsection{Measure}

At baseline, participants were asked to complete demographic information including year born, gender, race/ethnicity, level of education, years since TBI, years worked before TBI, years worked after TBI, and Facebook use. The knowledge assessment measure contained 10 multiple choice items concerning knowledge of evidence-based employment knowledge. The measurement instrument was used at both baseline and post-intervention. The number of items correct was used as baseline and postintervention score for each participant. An example of a knowledge question:

Which one of the following statements best describes the support that a job coach or employment specialist can provide if you are receiving supported employment services?

a) An employment specialist can provide job placement services. Once you are hired, supported employment services will be discontinued. 
b) An employment specialist will meet with you to identify your job interests and the supports that you will need to find and maintain a job. This includes supporting you through the interview process and supporting you on the job to learn the skills required to be successful.

c) An employment specialist can provide guidance and counseling services to help you develop your interview skills and develop a resume.

d) An employment specialist will meet with a number of employers on your behalf and find a job for you. Once you are hired, the employment specialist will negotiate on your behalf the conditions of employment.

\subsection{Data analysis}

Preliminary analysis consisted of examining frequencies, central tendencies, variance, and distributions. Chi-square, Fisher Exact test, independent $t$-tests, and Welch Modified Analysis of Variance (ANOVA) were used to determine whether there were significant differences between KT groups by personal demographics. Categories were collapsed when expectancy violations occurred when conducting chisquare tests. Analyses indicated unequal variance for years since TBI and number of jobs since TBI. Therefore, Welch Modified ANOVA was used for these two analyses. Repeated measures ANOVA was used to analyze research question one. Within and between groups effects are reported. Research question two was analyzed using independent $t$-test with gender and education as the independent variable.

\section{Results}

The two groups had similar gender composition with $53 \%(n=16)$ in the Facebook group being female and $73 \%$ being female in the e-news group $\left[\chi^{2}=2.12, p=0.15\right]$. There was no group difference in age $[t(56)=0.97, p=0.92]$ with the mean age in the Facebook group of $45.33(\mathrm{SD}=10.79)$ and 45.06 $(\mathrm{SD}=10.23)$ for the e-news group. Using two Fischer Exact Test analyses, the treatment and control groups were similar and predominantly White with 86\% being White in the Facebook group and 93\% of the e-news group being White when compared to the combined other racial/ethnic groups $(p=0.48)$. There was a statistically significant difference in the education level between the two groups when groups were collapsed into those who had "at least a bach-
Table 1

Frequencies of race/ethnicity and education level by group

\begin{tabular}{|c|c|c|c|}
\hline & & $\begin{array}{c}\text { Facebook } \\
\text { Group } \\
(n=30)\end{array}$ & $\begin{array}{c}\text { e-news } \\
\text { Group } \\
(n=30)\end{array}$ \\
\hline \multirow{5}{*}{$\begin{array}{l}\text { Race/ } \\
\text { Ethnicity }\end{array}$} & Asian & $1(3.3 \%)$ & $1(3.3 \%)$ \\
\hline & Hispanic & $1(3.3 \%)$ & $0(0 \%)$ \\
\hline & $\begin{array}{l}\text { Black or African } \\
\text { American }\end{array}$ & $2(6.7 \%)$ & $1(3.3 \%)$ \\
\hline & White & $24(80.0 \%)$ & $26(86.7 \%)$ \\
\hline & Did Not Report & $2(6.7 \%)$ & $2(6.7 \%)$ \\
\hline \multirow[t]{6}{*}{ Education } & Some High School & $2(6.7 \%)$ & $0(0 \%)$ \\
\hline & $\begin{array}{l}\text { High School Diploma } \\
\text { or GED }\end{array}$ & $2(6.7 \%)$ & $1(3.3 \%)$ \\
\hline & $\begin{array}{l}\text { Some College or } \\
\text { Vocational Training }\end{array}$ & $10(33.3 \%)$ & $8(26.7 \%)$ \\
\hline & Bachelor's Degree & $6(20.0 \%)$ & $13(43.3 \%)$ \\
\hline & Graduate Degree(s) & $6(20.0 \%)$ & $8(26.7 \%)$ \\
\hline & Did Not Report & $4(13.3 \%)$ & $0(0 \%)$ \\
\hline
\end{tabular}

Table 2

TBI and employment history group statistics

\begin{tabular}{llcccc}
\hline & Group & $n$ & Mean & SD & SE \\
\hline Number of Jobs Before TBI & e-News & 26 & 3.65 & 2.50 & 0.49 \\
& Facebook & 30 & 4.13 & 2.99 & 0.55 \\
Number of Jobs After TBI & e-News & 29 & 2.03 & 2.46 & 0.46 \\
& Facebook & 29 & 3.07 & 3.55 & 0.66 \\
Years since TBI & e-News & 24 & 9.96 & 6.31 & 1.29 \\
& Facebook & 17 & 16.35 & 12.10 & 2.93 \\
\hline
\end{tabular}

elor's degree" and "less than a bachelor's degree" $\left[\chi^{2}=4.36, p=0.04\right]$ with $70 \%$ of the e-news group having at least a bachelor's degree compared to $36 \%$ of the Facebook group. Table 1 provides information on race and ethnicity as well as education by KT group. There were no statistically significant differences between the Facebook and email groups using the independent variable years since TBI with $F_{\text {Welch }}(1,22.18)=3.98, p=0.06,95 \%$ CI [9.59, 15.63], number of jobs before TBI with $t(37)=0.65$, $p=0.52,95 \% \mathrm{CI}[-1.97,1.01]$, or number of jobs after TBI with $F_{\text {Welch }}(1,49.78)=1.66, p=0.20,95 \%$ CI [1.74, 3.36]. This information is presented in Table 2.

Participants were asked to report on the frequency of using Facebook including how often they used Facebook to network with other TBI survivors and find information on employment services and supports. This information is presented in Table 3. Fifty-six percent of the Facebook intervention group reported using Facebook daily. In comparison, 76\% of the e-news group reported using Facebook daily. This included individuals who used it one to two times a day, three to four times a day, and five or more times a day. Fifty percent of the participants in the Facebook group reported never using Facebook to find information on employment services and supports. Sixty-six 
Table 3

Frequency of Facebook use

\begin{tabular}{lcr}
\hline & Facebook Group $(n=30)$ & e-news Group $(n=30)$ \\
\hline How often do you go online and view/use Facebook? & & \\
I do not use Facebook & $6.7 \%(n=2)$ & $0 \%(n=0)$ \\
Rarely & $10.0 \%(n=3)$ & $6.7 \%(n=2)$ \\
1 to 2 times a month & $10.0 \%(n=3)$ & $3.3 \%(n=1)$ \\
3 to 4 times a month & $13.3 \%(n=4)$ & $13.3 \%(n=4)$ \\
1 to 2 times a day & $16.7 \%(n=5)$ & $40.0 \%(n=12)$ \\
3 to 4 times a day & $20.0 \%(n=6)$ & $16.7 \%(n=5)$ \\
5 or more times a day & $20.0 \%(n=6)$ & $20.0 \%(n=6)$ \\
Prefer not to say & $0 \%(n=0)$ & $0 \%(n=0)$ \\
No response & $3.3 \%(n=1)$ & $0 \%(n=0)$ \\
How often do you use Facebook to find information on & & \\
services/supports on employment? & & \\
Do not use Facebook for employment information & $50.0 \%(n=15)$ & $66.7 \%(n=20)$ \\
1 to 2 times a month & $30.0 \%(n=9)$ & $16.7 \%(n=5)$ \\
3 to 4 times a month & $3.3 \%(n=1)$ & $13.3 \%(n=4)$ \\
1 to 2 times a day & $6.7 \%(n=2)$ & $3.3 \%(n=1)$ \\
3 to 4 times a day & $6.7 \%(n=2)$ & $0 \%(n=0)$ \\
5 or more times a day & $0 \%(n=0)$ & $0 \%(n=0)$ \\
Prefer not to say & $3.3 \%(n=1)$ & \\
How often do you use Facebook to connect or network & & \\
with individuals with TBI? & & \\
I do not use Facebook to network & $16.7 \%(n=5)$ & $10.0 \%(n=3)$ \\
Rarely & $26.7 \%(n=8)$ & $16.7 \%(n=5)$ \\
Once a month & $13.3 \%(n=4)$ & $0 \%(n=0)$ \\
Twice a month & $3.3 \%(n=1)$ & $3.3 \%(n=1)$ \\
At least once a week & $23.3 \%(n=7)$ & $36.7 \%(n=11)$ \\
At least once a day & $10.0 \%(n=3)$ & $23.3 \%(n=7)$ \\
Multiple times a day & $3.3 \%(n=1)$ & $6.7 \%(n=2)$ \\
Prefer not to say & $0 \%(n=0)$ & $3.3 \%(n=1)$ \\
\hline
\end{tabular}

Table 4

Means and standard deviations for baseline and post-intervention knowledge assessment

\begin{tabular}{lccr}
\hline & $\begin{array}{c}\text { Baseline Mean } \\
(S D)\end{array}$ & $\begin{array}{c}\text { Post-Intervention } \\
\text { Mean }(S D)\end{array}$ & $n$ \\
\hline Group & & & \\
$\quad$ Facebook & $2.93(2.03)$ & $4.67(2.25)$ & 30 \\
$\quad$ e-News & $2.93(2.43)$ & $5.07(2.03)$ & 30 \\
Gender & & & \\
$\quad$ Females & $2.58(2.32)$ & $4.52(2.37)$ & 38 \\
$\quad$ Males & $3.57(1.63)$ & $5.43(1.80)$ & 21 \\
Education & & & \\
$\quad$ College Graduate & $3.09(2.21)$ & $5.21(2.09)$ & 33 \\
$\quad$ Some College/Vocational Training & $3.00(2.06)$ & $4.39(2.20)$ & 18 \\
$\quad$ High School Graduate or Less & $2.29(2.21)$ & $4.57(2.37)$ & 7 \\
\hline
\end{tabular}

percent of the e-news group never used Facebook for employment information. In contrast, $50 \%$ of the Facebook group never used Facebook for this purpose. When asked how often did they use Facebook to connect or network with other TBI survivors, $65 \%$ of the e-news group used Facebook to network at least once a week or more often. In comparison, $35 \%$ of the Facebook intervention group used Facebook for this purpose at least once a week or more often.

The repeated measures ANOVA indicated a significance within group difference between baseline and post-intervention knowledge gain with
$F(1,58)=35.22, p=0.0001,95 \% \mathrm{CI}[-2.58,-1.28]$ and had a large effect size $(d)$ of 0.91 . No significant knowledge differences were found at postintervention between the Facebook group and e-News group with $F(1,58)=0.38, p=0.542,95 \% \mathrm{CI}[-0.71$, 1.51]. There were no significant differences in knowledge at post-intervention by gender $[t(57)=1.56$, $p=0.123$ ] or education $(1-$ high school graduate or less, 2- some college/vocational training, 3- college graduate or not $)[F(2,55)=0.931, p=0.400]$. See Table 4 for means and standard deviations for all comparisons. 


\section{Discussion}

The idea of individuals with TBI being able to access evidenced-based information on employment through Facebook is very timely. Individuals with TBI can become very isolated immediately after injury when many preinjury social connections have been disrupted. Family members, previous coworkers, and people in the community often are unsure about how to interact with someone who has had a significant injury such as a TBI. Facebook may offer a way to ameliorate some of this initial distancing. Individuals with TBI face a number of challenges in returning to work post trauma. Having knowledge of and using evidence-based employment practices may be one way to impact their ability to return to work. This study compared two different KT strategies to increase the knowledge of employment evidencebased practices of individuals with TBI.

While this study did not find that the use of Facebook as a KT strategy is more effective in increasing the employment knowledge of the participants, a significant within group difference between baseline and post-intervention knowledge gain was found. Participants did increase their employment knowledge from participation in this study. There are a number of advantages to using Facebook as well as e-news blasts to reach individuals with TBI. First, the participants can access the information on their own schedule. Once resources are posted in the Facebook group, they remained available. Once e-news blasts were sent out, participants could access the links or resources at their convenience. As an example, the "seen by" counter on Facebook provided documentation that individuals reviewed the posts several days to weeks after the initial date of posting.

The benefits of networking with other individuals with TBI through Facebook and establishing a sense of community could also be invaluable to individuals who have lost access to pre-injury social networks. However, a limitation of this study may have been that the participants were unknown to each other when the secret group was established. Typically, Facebook is used as a tool for friends and acquaintances to communicate with each other. There were limited information exchanges between the participants during the course of the three month intervention and most exchanges were facilitated by the peer mentor and lead researcher. The impact of being uncomfortable with unfamiliar individuals also may have been a factor in the number of participants who actually posted in the group on a regular basis. One strategy to consider for future research may be to conduct focus group meetings prior to beginning the study and at ongoing intervals such as once a month during the intervention. The meetings could occur using the telephone or a virtual meeting software program that provides video as well as audio access for participants. These real-time, synchronous discussions could serve several purposes: 1) identify the information needs of the participants prior to beginning the project, and 2) provide ongoing feedback to the researchers on the format and usefulness of the information.

The effects that a moderate to severe TBI may have on an individual's return to work also may have impacted the outcome of this study. Cognitive deficits resulting from a TBI may include but are not limited to memory challenges, attention and concentration, processing and understanding information, learning new information, communication, planning and organizing, and problem-solving [12]. In addition, the severity of these challenges varies from individual to individual based on the severity of the disability, and an individual with a TBI may not experience all of these challenges. In a study conducted by Tsaousides and colleagues, individuals with TBI reported cognitive deficits, such as remembering computer procedures or understanding instructions, as a deterrent to using Facebook [11]. The findings from this study seem to support this report.

Attrition was a concern in this study and highlights the need to consider ways to address the issue such as having a larger sample size for future research. However, attrition also may have been impacted by the memory challenges faced by individuals with TBI. As an example, of the 138 individuals who consented to participate, 49 individuals never took the pretest after completing the online consent process even though three follow-up email reminders were sent. One individual who consented to participate asked to be withdrawn from the study saying that she did not know anything about the study even though she had consented to participate. Problems with attrition also occurred at the end of the study when requests were sent for the participants to complete the posttest. After three email reminders, 18 of the study participants still had not taken the posttest. At that point, a followup telephone call was added to the research protocol to remind the participants to complete the posttest as well as ask if they needed assistance. This procedure resulted in 11 additional participants completing the posttest. Seven participants still failed to take the posttest even with this added accommodation. One 
of the seven individuals was an active member of the Facebook group and adamantly reported that he remembered taking the posttest when called by one of the researchers. Since all communications were sent via email except for one follow-up telephone reminder, future studies should consider alternative forms of communication such as telephone contact, postcard reminders, Tweets, text messages, or other methods of communication. A larger sample size may have provided more data to determine if either strategy was more effective in increasing the employment knowledge of the participants.

Failure to follow the Facebook posts on a regular basis may have contributed to the limited increase in knowledge by some participants assigned to that group. During a follow-up focus group with participants, one group agreed that they often forgot about the group and needed additional reminders to participate. The researchers assumed that participants would be reminded to view the group page when emails were sent through the notifications feature on Facebook alerting that a new post was added. However, these notifications or "natural cues" may not have been sufficient to prompt the participants to visit the Facebook page and read the new information. Using the "seen by" feature on the posts, the researchers were able to review how many participants saw the post, as well as determine how many participants actually commented. When the lead researcher, who was also a member of the group, asked why participants did not comment on more of the group posts, several participants responded that they were uncomfortable with responding due to their limited ability to write a response. Determining the compensatory strategies as well as accommodations that assist individuals with TBI in participating and understanding the information provided is clearly something that needs to be addressed in future research.

Individuals with more significant challenges resulting from TBI may benefit from the assistance of an advocate who helps them identify compensatory strategies to facilitate participation. Involving a participant's VR counselor or employment specialist in providing needed assistance in accessing the group information may be one strategy to consider for future research. For instance, the advocate could be involved in the early stages of learning to use Facebook and then gradually fading assistance as the person becomes more independent. This is much like the assistance that is provided by an employment specialist when first assisting an individual learning a new job. Intensive supports are provided initially including identifying strategies that help the person become independent. Then, that assistance is gradually faded as the person no longer requires the assistance.

The length of the study also may have impacted the knowledge gains for both groups, since the intervention spanned only three months. For instance, the e-news group received one email contact with information once a month for a total of three contacts during the study. There was virtually no communication between the researchers and the participants who received the e-news blasts even though the emails encouraged the participants to ask questions. Only one participant replied to an email saying that he found the information too complicated to understand. While there were a minimum of five posts per week during this time period in the Facebook group, most of these posts were brief consisting of one to two paragraphs. The cumulative amount of information may not have been sufficient to demonstrate social media as the most effective way to impact the knowledge of the participants. In addition, most of the posts were written communication with limited information provided in other formats such as audio or video. Future researchers should consider the format (video, pictures, words) that would be most effective in in increasing participant knowledge.

While this study paired a peer mentor with a content expert, it does not provide any data on whether this approach is more effective in assisting individuals in learning new knowledge and then acting on that knowledge. During follow-up focus group discussions, participants expressed that they liked having the mentor as the group facilitator. Participants also consistently asked the mentor to provide feedback on his experiences that would be similar to their own when seeking employment. Future research is needed to determine if this is an essential factor in increasing participant knowledge.

In conclusion, this research provides a good pilot study on the use of Facebook to impact the employment knowledge of individuals with TBI. As social interaction and information distribution continue to occur more commonly in online networking formats, it is important to consider whether access can be equally achieved by all users, including individuals with TBI. This study has provided valuable information on the use of Facebook as one KT strategy to promote the use of knowledge by individuals with TBI. Future research will be guided by this information to determine if in fact social media can increase the use of evidence-based research on employment. 
Questions to be answered include: 1) Does adding additional cues and compensatory strategies to the existing Facebook features facilitate the use of social media by individuals with TBI (e.g., Tweets, text messages, specialized apps, etc.)? 2) Are the unique challenges faced by individuals with TBI in accessing Facebook the same or different for individuals with other disabilities (spinal cord injury, multiple sclerosis, cerebral palsy, cognitive disabilities, etc.)? 3) Would increasing the length of the intervention impact the knowledge gains and use of evidencebased employment practices? 4) Does increased knowledge of evidence-based employment practices facilitate use and impact the employment outcomes of individuals with TBI?

\section{Acknowledgments}

This study was supported by the Center on Knowledge Translation for Employment Research through grant 90DP0009 (formerly ED \#H133A100026) to SEDL, an affiliate of American Institutes for Research, from the National Institute on Disability, Independent Living, and Rehabilitation Research (NIDILRR) in the U.S. Department of Health and Human Services (HHS), Administration for Community Living. However, the contents of this article do not necessarily represent the policy of the U.S. Department of HHS, and you should not assume endorsement by the Federal Government.

\section{Conflict of interest}

None to report.

\section{References}

[1] Centers for Disease Control and Prevention. Report to Congress on traumatic brain injury in the United States:
Epidemiology and rehabilitation [Internet]. Atlanta, GA: National Center for Injury Prevention and Control; Division of Unintentional Injury Prevention; 2015 [cited 2016 Jul 26]. Available from: https://www.cdc.gov/traumaticbraininjury/ pdf/tbi_report_to_congress_epi_and_rehab-a.pdf

[2] Van Velzen JM, Van Bennekom CA, Edelaar MJ, Sluiter JK, Frings-Dresen MH. How many people return to work after acquired brain injury? A systematic review. Brain Inj 2009;23(6):473-88.

[3] Shames J, Treger I, Ring LI, Giaquinto S. Return to work following traumatic brain injury: Trends and challenges. Disabil Rehabil 2007;29(17):1387-95.

[4] Inge KJ, Graham CW, Erickson D, Sima A, West M, Cimera RE. Improving the employment outcomes of individuals with traumatic brain injury: The effectiveness of knowledge translation strategies to impact the use of evidence-based practices by vocational rehabilitation counselors. J Vocat Rehabil 2016;45(1):107-15.

[5] Wehman P, Targett P, West M, Kregel J. Productive work and employment for persons with traumatic brain injury: What have we learned after 20 years? J Head Trauma Rehabil 2005;20:115-27.

[6] Smith C. By the numbers: 200+ amazing Facebook statistics [Internet]. United States: DMR; 2016 [cited 2016 March]. Available from: http://expandedramblings.com/index.php/ by-the-numbers-17-amazing-facebook-stats/

[7] Protalinski E. Facebook passes 1.44B monthly active users and 1.25B mobile users; $65 \%$ are now daily users [Internet]. United States: Venture Beat; 2015 April [cited 2016 April]. Available from: http://venturebeat.com/2015/ 04/22/facebook-passes-1-44b-monthly-active-users-1-25bmobile-users-and-936-million-daily-users

[8] D'Onfro J. Here's how much time people spend on Facebook per day [Internet]. United States: Business Insider; 2015 Jul [cited 2016 April]. Available from: http:// www.businessinsider.com/how-much-time-people-spendon-facebook-per-day-2015-7\#ixzz3fKtewifD

[9] Olmstead K, Lampe C, Ellison NB. Social media and the workplace [Internet]. United States: Pew Research Center; 2016 June [cited $2016 \mathrm{Jul}$ ]. Available from: http://www.pew internet.org/2016/06/22/social-media-and-the-workplace/

[10] Shpigelman CN, Gill CJ. Facebook use by persons with disabilities. J Comput Mediat Commun 2014;19(3):610-24.

[11] Tsaousides T, Matsuzawa Y, Lebowitz M. Familiarity and prevalence of Facebook use for social networking among individuals with traumatic brain injury. Brain Inj 2011:25 (12):1155-62

[12] Neumann D, Lequerica A. Cognitive problems after traumatic brain injury [Internet]. United States: BrainLine.Org; 2002 [cited 2016 April]. Available from: http://www.brain line.org/content/2010/03/cognitive-problems-after-trauma tic-brain-injury_pageall.html 